ARTICLE

https://doi.org/10.1038/s41467-019-13013-8

\title{
Conditional quorum-sensing induction of a cyanide-insensitive terminal oxidase stabilizes cooperating populations of Pseudomonas aeruginosa
}

Huicong Yan ${ }^{1,2}$, Kyle L. Asfahl (10 ${ }^{3}$, Na Li, ${ }^{1,2}$, Feng Sun ${ }^{1,2}$, Junwei Xiao ${ }^{1,2}$, Dongsheng Shen ${ }^{1,2}$, Ajai A. Dandekar (1) ${ }^{3,4 \star} \&$ Meizhen Wang (1) ${ }^{1,2 \star}$

Pseudomonas aeruginosa, an opportunistic pathogen of humans, uses quorum sensing (QS) to regulate the production of extracellular products that can benefit all members of the population. P. aeruginosa can police QS-deficient cheaters by producing hydrogen cyanide, which is also QS regulated; however, the mechanism by which cooperators selectively protect themselves from the toxicity of cyanide remained unresolved. Here, we show that a cyanideinsensitive terminal oxidase encoded by cio $A B$ provides resistance to cyanide, but only in QSproficient strains. QS-deficient cheaters do not activate cioAB transcription. QS-mediated regulation of $c i O A B$ expression depends on production of both cyanide by cooperators (which is QS regulated) and reactive oxygen species (ROS) from cheaters (which is not QS regulated). This type of regulatory system allows cooperating populations to respond, via ROS, to the presence of cheaters, and might allow them to defer the substantial metabolic cost of policing until cheaters are present in the population.

\footnotetext{
${ }^{1}$ School of Environmental Science and Engineering, Zhejiang Gongshang University, Hangzhou, China. ${ }^{2}$ Zhejiang Provincial Key Laboratory of Solid Waste Treatment and Recycling, Hangzhou, China. ${ }^{3}$ Department of Medicine, University of Washington, Seattle, WA, USA. ${ }^{4}$ Department of Microbiology, University of Washington, Seattle, WA, USA. *email: dandekar@u.washington.edu; wmz@zjgsu.edu.cn
} 
T he opportunistic pathogen Pseudomonas aeruginosa uses a complex QS circuit to regulate the production of numerous shared or public goods, including secreted proteases and other virulence factors ${ }^{1,2}$. The QS circuitry in $P$. aeruginosa consists of two acyl-homoserine lactone (AHL) signal-receptor pairs. In the las system, the signal synthase LasI produces a signal, $\mathrm{N}$-3-oxo-dodecanoyl homoserine lactone (3OC12-HSL), which binds to the transcription factor LasR. In the second, the rhl system, the signal synthase RhlI produces $N$-butyryl-homoserine lactone (C4-HSL), which is bound by the transcription factor RhlR. The LasI-R system regulates the RhlI-R system, so null mutations in LasR have the effect of inactivating both QS systems ${ }^{1,2}$. When $P$. aeruginosa is passaged in conditions where QS is required to obtain carbon and energy for growth, such as in media containing the protein casein as the sole carbon and energy source, social cheaters reproducibly emerge in the population ${ }^{3,4}$. These social cheaters invariably have LasRinactivating mutations $\mathrm{s}^{3,4}$.

In microbial communities, the most beneficial strategies for individual cells can be at odds with the best strategy for the entire population. Cooperating individuals may produce a costly public good that can be shared and serve to enhance fitness of the entire population. However, selection often favors cheating individuals that avail themselves of public goods while avoiding investment, ultimately risking cooperative collapse ${ }^{5,6}$. Several mechanisms have been described to stabilize cooperation in populations, including delayed production of public goods ${ }^{7}$, and independent regulation of multiple public goods 8 .

We have shown that $P$. aeruginosa cooperators secrete hydrogen cyanide (HCN) as a means to "police" cheaters9. Policing depends on activation of the RhlI-R QS circuit ${ }^{9}$, which regulates the genes that code for production of the hydrogen cyanide synthase ${ }^{10}$. Deletion of either RhlR or the genes that encode the cyanide synthase results in a loss of policing by cooperators. In policing, pleiotropic regulation of both toxin and immunity mechanisms allows a cooperating population to punish neighboring cells that lack sufficient relatedness at a specific locus ${ }^{11}$. Without this policing mechanism, the cheaters outcompete cooperators and cooperating populations collapse, as there is ultimately an insufficient number of cooperators to continue growing in conditions where QS is required for growth, a type of "tragedy of the commons".

Policing comes at a cost to the cooperators: in pure cultures, bacteria that produce cyanide grow more slowly and to a lower yield than bacteria that do not make cyanide ${ }^{9}$. It is not apparent from previous studies of $P$. aeruginosa QS how cooperators might protect themselves against cyanide intoxication. The $P$. aeruginosa genome contains a conserved cyanide-insensitive terminal oxidase that, unlike hydrogen cyanide synthase, has not been shown to be upregulated in published QS transcriptomes ${ }^{12,13}$. We are interested in the mechanism of self-protection against cyanide intoxication by cooperating populations of $P$. aeruginosa.

Here, we show that QS-proficient bacteria are more resistant to death in the presence of cyanide than QS-deficient cheaters. We further demonstrate this difference in survival reflects conditional quorum regulation of genes encoding a cyanide-insensitive terminal oxidase, $\operatorname{cio} A B$.

\section{Results}

Cheaters are more sensitive to cyanide than cooperators. Initially, we asked if cooperators could better survive cyanide intoxication than cheaters, by comparing the resistance either of the cooperators or the cheaters to exogenously added cyanide. We grew wild-type (WT) P. aeruginosa PAO1, or a LasR-null mutant of PAO1, in LB-MOPS broth with a range of added potassium cyanide $(0-500 \mu \mathrm{M})$, which mimics the maximal concentration of cyanide ion $\left(\mathrm{CN}^{-}\right)$produced by PAO1 in stationary phase ${ }^{14}$. After $16 \mathrm{~h}$, survival of WT PAO1 under $100 \mu \mathrm{M}$ of $\mathrm{CN}^{-}$stress was 3.5-fold higher than for LasR-null mutants (Fig. 1a); the median lethal concentration $\left(\mathrm{LC}_{50}\right)$ of $\mathrm{CN}^{-}$was $\sim 470 \mu \mathrm{M}$ for the WT and $60 \mu \mathrm{M}$ for LasR mutants, respectively (Fig. 1a).

Hydrogen cyanide inhibits cytochrome $\mathrm{C}$ oxidase in susceptible cells, resulting in production of reactive oxygen species (ROS) ${ }^{15,16}$. Therefore, we reasoned that ROS production should be greater from a population of LasR-null mutants than the WT, when grown in the presence of cyanide. To measure the generation of ROS, we used a fluorescence assay as described in "Materials and Methods". ROS production from a population of LasR-mutants in the presence of $150 \mu \mathrm{M} \mathrm{CN}^{-}$was 2.2 -fold greater than in the absence of $\mathrm{CN}^{-}$(Fig. 1b). WT populations showed very little increase in ROS production when exposed to $\mathrm{CN}^{-}$. Resistance to another generator of reactive oxygen species, $\mathrm{H}_{2} \mathrm{O}_{2}$, was also higher in WT PAO1 than in LasR-mutants (Fig. 1c).

We next asked if the outcome of competition between WT PAO1 and LasR-null mutants would be affected by the addition of catalase, as addition of catalase can protect against ROS damage by converting peroxide to water and oxygen. When the WT and LasR-mutants are passaged in casein broth, the LasRmutants and WT come to an equilibrium of $\sim 20 \%$ cheaters $^{3,4,9}$ (Fig. 1d). However, when 200 units catalase were added to the culture daily with passage, cooperators lost the ability to police cheaters, which came to a high percentage in the population (Fig. 1d). At this point, the population could no longer be propagated for lack of sufficient numbers of cooperators.

QS activation alone is insufficient to confer cyanide resistance. A survival difference between the WT and LasR-mutants in the presence of cyanide, along with evidence for the regulation of cyanide production by $\mathrm{RhlR}^{9}$, implies that the mechanism of cyanide resistance is QS-regulated. Three mechanisms, each involving different gene products, have been reported to ameliorate cyanide toxicity in $P$. aeruginosa ${ }^{17-19}$. However, none of these gene products are known to be QS-regulated ${ }^{12,13}$. One of the gene products, the enzyme Cio, a cyanide insensitive terminal oxidase encoded by $\operatorname{cio} A B$, allows $P$. aeruginosa to respire oxygen in the presence of cyanide. Complementing the function of Cio are the gene products of a six-gene cluster, PA4129-4134, that synergizes with Cio through an unknown mechanism to protect against cyanide intoxication. Finally, the protein RhdA, a cyanide sulfur-transferase, can convert cyanide to thiocyanate, which is less toxic.

Previous reports about QS-regulated genes in $P$. aeruginosa have not identified the $\operatorname{cio} A B$, rhdA or PA4129-4134 genes as RhlR- or LasR-regulated ${ }^{12,13}$. A possible explanation for this paradox would be that regulation of one or all depends on the presence of cyanide. To determine which of these gene products might be contributing to the cyanide resistance phenotype, we measured transcript levels of cioA (PA3930), rhdA (PA4956), and PA4133 (in the six gene cluster) in WT PAO1 in the presence and absence of $150 \mu \mathrm{M}$ potassium cyanide. In the presence of cyanide, the expression levels of cioA and PA4133 were about 7-fold and 14-fold higher in WT PAO1 than in the absence of cyanide, suggesting a possible role for both gene products in protection against cyanide intoxication (Fig. 2a). Expression levels of $r h d A$ were too low to be detected in any of the conditions tested. These results were consistent with the idea that the presence of cyanide was a condition required for the expression of resistance genes.

We next asked if QS was also required for induction of either cioA or PA4133. We measured transcript levels of both of these 

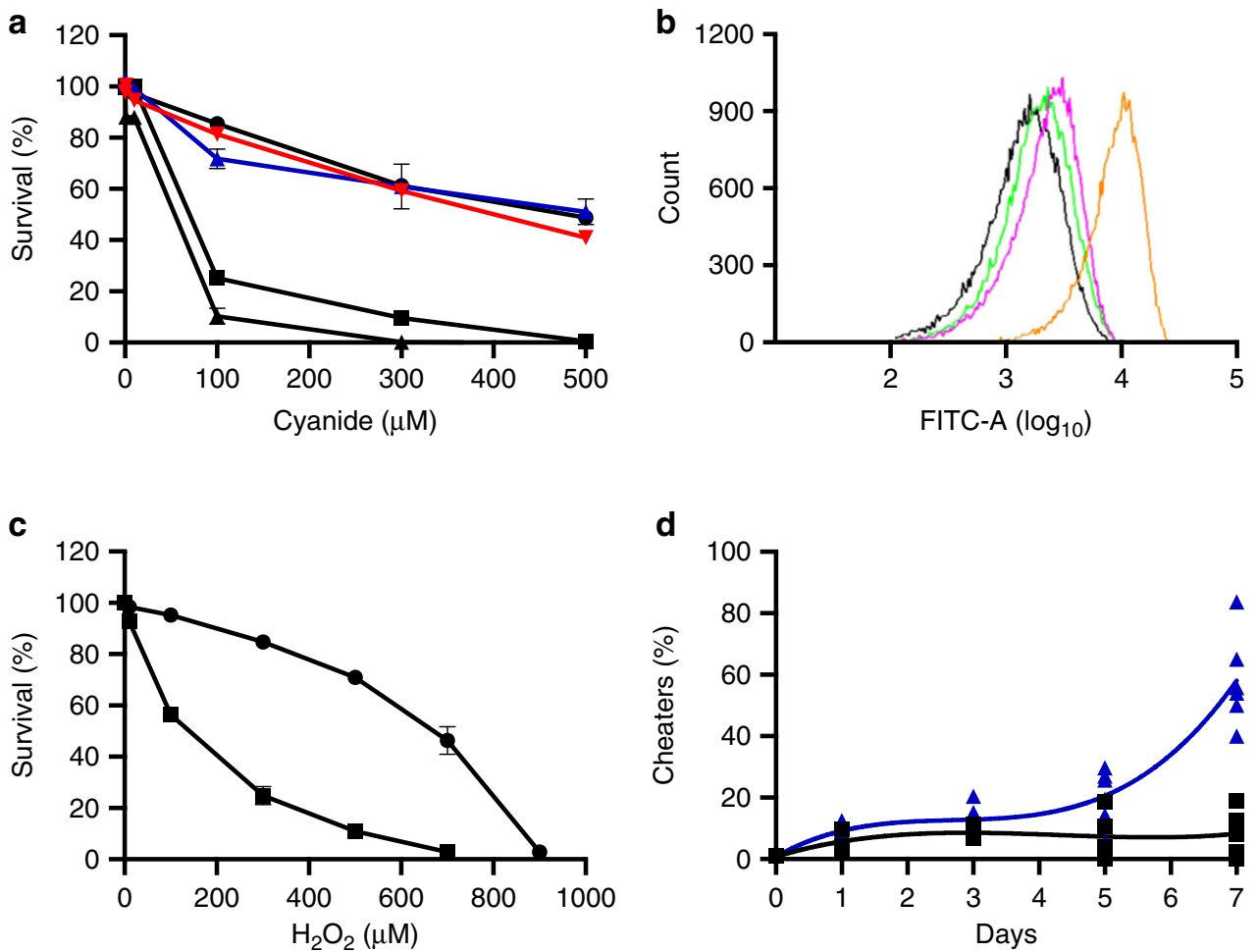

Fig. 1 Wild-type PAO1 is more resistant to cyanide and ROS intoxication than a LasR mutant. a Survival of WT PAO1, LasR-mutant, RhdA-mutant, PA4133mutant, or CioA-mutant, grown in the presence of different concentrations of cyanide with initial $\mathrm{OD}_{600}$ of 0.005 . The cell density was measured after $16 \mathrm{~h}$ of growth. Black circles, WT PAO1; red inverted triangles, RhdA-mutant; blue triangles, PA4133-mutant; black squares, LasR-mutant; black triangles, CioAmutant. b ROS production from the WT PAO1 and LasR-mutant when grown in LB-MOPS with or without $150 \mu \mathrm{M} \mathrm{CN}^{-}$for 40 min. ROS generation was measured by FTC fluorescence. Black, WT PAO1 without cyanide; green, WT PAO1 with cyanide; pink, LasR-mutant without cyanide; orange, LasR-mutant with cyanide. c Survival of WT PAO1 or LasR-mutants in the presence of hydrogen peroxide after $16 \mathrm{~h}$ of growth. Circles, WT PAO1; squares, LasR-mutants. d Cheater frequency in the presence or absence of catalase. The initial frequency of cheaters was 0.01. Black, competition without catalase; blue,

competition with $25 \mathrm{U} / \mathrm{mL}$ of catalase added daily. All experiments were performed with a minimum of three biological replicates. Lines represent best-fit polynomial regressions for each condition. For a, c means, s.e.m., and individual biological replicates are shown and in some cases error bars are too small to be seen

genes in WT PAO1 as compared with LasR-RhlR-double mutants, and found that the QS-proficient WT strain had eightfold more cioA transcript and 17-fold more PA4133 transcript than the QS-deficient strain (Fig. 2a), in the presence of cyanide. Together with the differential susceptibility of each of these strains to cyanide (Fig. 1a), the results suggested to us that self-protection against cyanide policing is in part conditional on the policing mechanism itself or the ecological consequences of policing within a mixed population.

One alternative explanation for these results is that Cio expression is dependent on the intracellular production of cyanide, not QS activation per se. That is, because cyanide is produced at very low levels in LasR-null mutants ${ }^{20,21}$, it is possible that the protection conferred by Cio is not activated in these strains as those cells do not make cyanide. To determine if endogenously-produced cyanide could activate Cio (or other protective mechanisms) in the LasR-null mutant, we introduced an additional copy of the $h c n A B C$ operon at neutral site on the chromosome of both WT and LasR-null mutants, with the genes under control of the arabinose-inducible ParaBAD promoter. Addition of arabinose to cells harboring this construct results in QS-independent cyanide production. We competed these strains against each other in casein broth supplemented with Larabinose. The LasR mutant fared more poorly in competition when arabinose was added (Supplementary Fig. 1), which indicates that endogenous cyanide production in a LasR mutant does not activate the cyanide protection mechanism. cio $A B$ is upregulated in cooperators when cheaters are present. We reasoned a second potential explanation for the conditional regulation of self-protection to cyanide could be response to the culture conditions where the population is actively policed. To test this idea, we measured gene expression in the context of cocultures between two different types cooperators and cheaters. In one configuration, WT PAO1 should police LasR-mutants through the production of cyanide (Fig. 1d) (a "policing" condition) $)^{3,4,9}$. In another, a RhlR-null cooperator cannot police a RhlR-LasR-double mutant cheater (a "non-policing" condition) ${ }^{9}$. We separated cheaters and cooperators in these experiments with dialysis membranes and compared expression by cooperators of cioA, PA4129, PA4131, PA4133, or rhdA in both the "policing" and "non-policing" conditions. We added analysis of PA4129 and PA4131 in this analysis because differences in PA4133 expression were markedly different than that seen in the presence/absence of cyanide (Fig. 2a). There were no significant differences in expression of PA4129, PA4131, PA4133, or rhdA between the policing and non-policing conditions (Fig. 2b). However, expression of $c i o A$ in the policing condition was nearly 50 times the level observed in the non-policing condition. Together, these data are consistent with the hypothesis that $\operatorname{cio} A B$ expression is the relevant factor in QS-mediated protection against selfintoxication, and that QS regulation of these genes depends on the QS-regulated production of cyanide in the conditions of our experiments. This is a type of AND logic gate: gene regulation depends on a QS transcription factor and also an environmental 

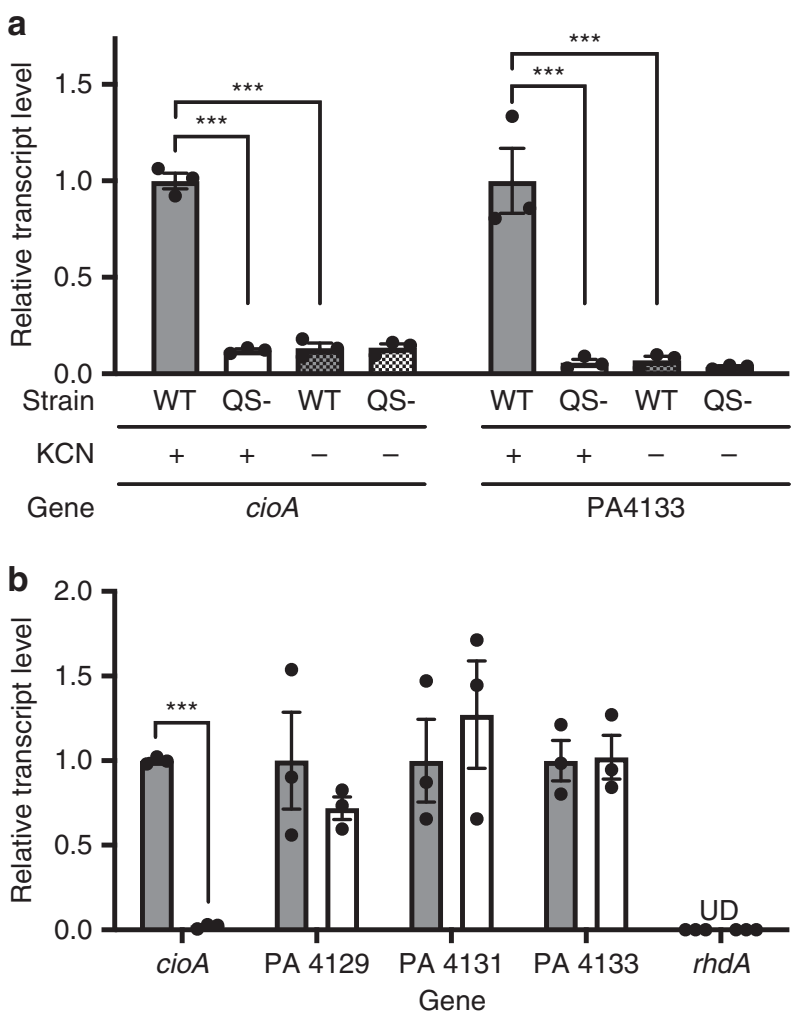

Fig. 2 Regulation of cioA (PA3930) by QS is conditional on the presence of cyanide. a Expression of cioA (PA3930) and PA4133 in the WT PAO1 (black, "WT") and LasR-RhIR-double mutant (white, "QS-"). Solid, in the presence of $150 \mu \mathrm{M} \mathrm{KCN}$; grid, in the absence of $150 \mu \mathrm{M} \mathrm{KCN}$. b cioA, PA4129, PA4131, PA4133, and rhdA expression from either WT PAO1 ("policing", black) or RhIR-null cooperators ("non-policing", white) when grown in co-culture with cheaters, with the populations separated by dialysis membranes. Expression was measured by RT-PCR with proC used as reference gene. Means, s.e.m., and individual biological replicates are shown $(n=3)$. Statistical significance determined by unpaired Student's $t$-test; ${ }^{\star \star \star} p<0.001$. UD, undetected

condition or cue ${ }^{22}$, and in this case the cue is likely environmental ROS.

Our earlier observation that a CioA-null mutant was more sensitive to cyanide intoxication than WT PAO1 (Fig. 1a) is consistent with the idea that Cio plays an important role in policing. To ascertain if the CioA-mutants lost the ability to police cheaters, by virtue of no longer having an advantage over LasR-mutants in terms of cyanide tolerance, we grew CioAmutants in co-culture with LasR-mutants at a starting ratio of 99:1. CioA-mutants are known not to have a defect in cyanide production ${ }^{17}$. Within 3 days, the cheater proportion increased to about 50\% (Fig. 3a). By contrast, both WT PAO1 and the PA4133-null mutants were able to police cheaters and the frequency plateaued at $20 \%$, as previously described ${ }^{3,4,9}$. Although the CioA-null co-culture could not be propagated past day 5 for lack of sufficient cooperators, there was no difference in population productivity of QS-regulated exoproducts compared with WT PAO1, as measured by total elastase and cyanide production by CioA-null or PA4133-null cooperators (Supplementary Fig. 2). None of these deletions adversely affect the growth rate or final cell density in either LB-MOPS or casein broth (Supplementary Fig. 3). We also did not observe differences in either the expression of lasB, lasR, or rhlR (Fig. 3b) or the production of public good elastase (Fig. 3c) between these three strains. These results indicated that Cio, not the gene products of
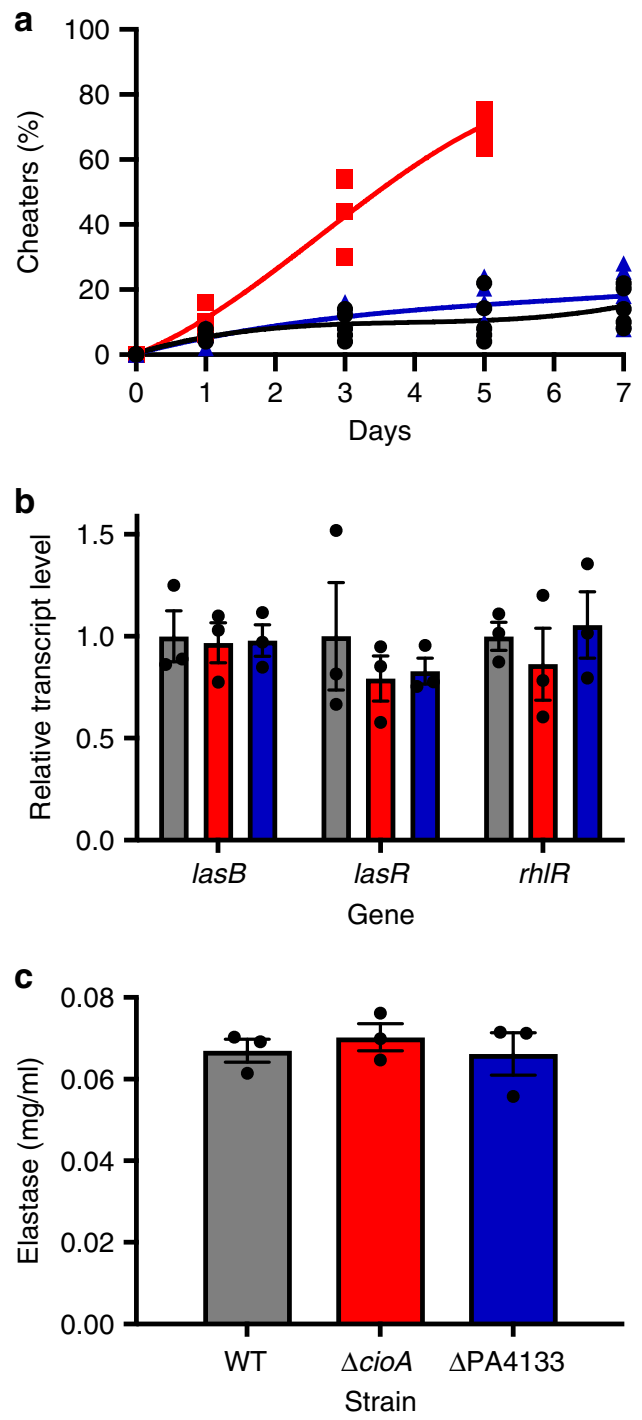

Fig. 3 The cyanide insensitive terminal oxidase Cio mediates self-protection of cooperators. a Competition between WT PAO1 (black), CioA-null cooperator (red), or PA4133-null cooperator (blue) and LasR-null mutants. The initial frequency of cheaters was 0.01 . Five biological replicates were performed. Lines represent best-fit polynomial regressions for each condition. b Expression of lasB, lasR and rhIR in WT PAO1 (black), CioA-null mutant (red), and PA4133-null mutant (blue). c Elastase production by WT PAO1 (black), CioA-null mutant (red), and PA4133-null mutant (blue). For b, c means, s.e.m., and individual biological replicates are shown $(n=3)$

PA4129-4134, is the critical element of QS-regulated selfprotection from cyanide intoxication in the conditions of our experiments.

Interference with CioA prevents policing. Because a CioA-null cooperator is susceptible to a tragedy of the commons (Fig. 3a), we reasoned that interfering with the expression of Cio in strain PAO1 could increase the frequency of QS-deficient cheaters, resulting in a failure to propagate. To do so, we added copper (in the form of copper sulfate) to the growth medium, which has been reported to decrease $\operatorname{cio} A B$ expression while also stabilizing the non-Cio high-affinity aerobic terminal oxidases ${ }^{23}$. The predicted effect of copper sulfate addition is twofold: cooperators would have less Cio, and stabilization of terminal oxidases that would otherwise be poisoned by cyanide might protect cheaters from policing. 

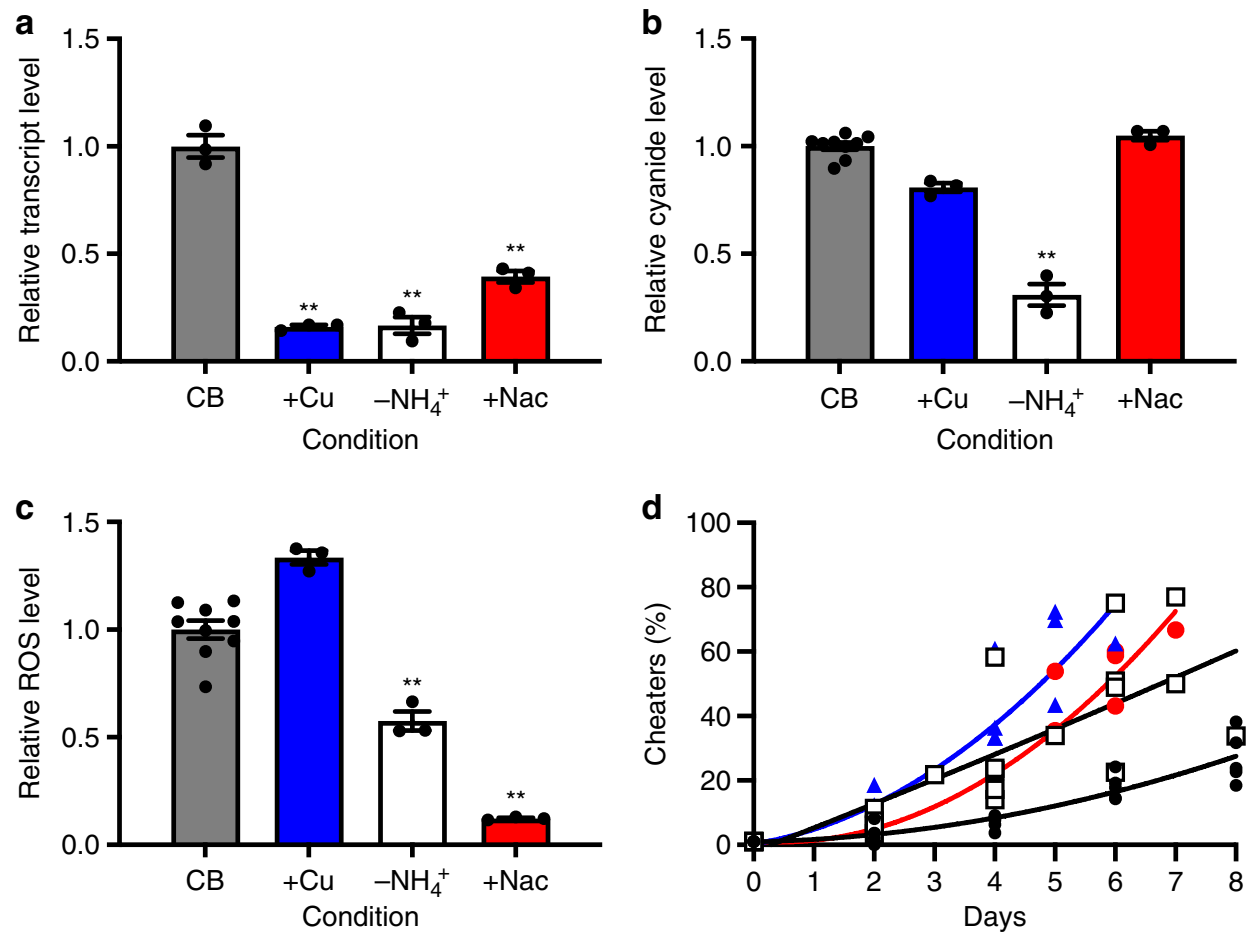

Fig. 4 Inhibition of cio $A$ expression as a potential strategy to destabilize populations of $P$. aeruginosa. Expression of $\mathbf{a}$ cio $A$ and $\mathbf{b}$ production of cyanide when WT PAO1 is grown in casein broth (CB, black) with the addition of $1 \mathrm{mM}$ copper sulfate $(+\mathrm{Cu}$, blue). Shown in comparison are casein broth without ammonium sulfate $\left(-\mathrm{NH}_{4}{ }^{+}\right.$, white), and media with added $\mathrm{N}$-acetylcysteine ( $+\mathrm{Nac}$, red). c ROS levels in the same conditions. $\mathbf{d}$ Cheater frequency when WT cooperators and LasR mutants are grown in these conditions. In all co-cultures, the starting ratio of WT:LasR mutants was 99:1. At least three biological replicates were performed. Lines represent best-fit polynomial regressions for each condition. For a-c, means, s.e.m., and individual biological replicates are shown. Statistical significance determined by paired Student's $t$-test versus CB alone; ${ }^{\star \star} p<0.01$. UD; undetected

We first tested the effect of copper sulfate addition to casein broth in monocultures of WT PAO1 and found a reduction in expression of cioA nearly as much as if ammonia, a necessary substrate for hydrogen cyanide synthesis ${ }^{24}$, was not present (Fig. 4a). This finding was not paralleled by a decrease in cyanide synthesis, where absence of ammonium sulfate decreases cyanide production significantly compared with the control (Fig. 4b) ${ }^{24}$. These data show that addition of copper sulfate decreased cioA expression in cooperators but had little or no effect on cyanide production. Addition of $1 \mathrm{mM} \mathrm{N}$-acetylcysteine, an ROS scavenger, did not reduce cioA expression to the same degree as copper sulfate and, similar to copper sulfate addition, did not affect cyanide production (Fig. 4a, b).

We then measured ROS generation in cultures of WT PAO1 treated with copper sulfate, $\mathrm{N}$-acteylcysteine, or grown in the absence of ammonium sulfate. ROS production was significantly reduced in both ammonium-sulfate-free media or when $\mathrm{N}$ acetylcysteine was added (Fig. 4c). Addition of copper sulfate, which reduced cioA expression, had an insignificant impact on hydrogen cyanide production and therefore ROS generation (Fig. 4b, c).

Finally, we asked if LasR-mutant cheaters could increase to an unsustainable frequency in copper-sulfate treated populations, reasoning that interference with $c i o A$ expression may produce a similar effect as observed in competitions with a CioA-null mutant (Fig. 3a). We grew WT PAO1 and LasR-mutants together in casein broth at a starting ratio of 99:1. Again, because of cyanide policing, the LasR mutants come to an equilibrium with the wild-type PAO1 at a frequency of $20-40 \%$ (Fig. 4d). Consistent with our hypothesis, the addition of copper sulfate resulted in a dramatic increase in the frequency of LasR-mutant cheaters, ultimately exceeding $60 \%$ with a concomitant tragedy of the commons where the population could no longer be propagated. We observed a similar consequence when $\mathrm{N}$ acetylcysteine was added to or ammonium sulfate was absent from the growth medium, consistent with prior reports ${ }^{24}$.

\section{Discussion}

Maintenance of cooperation in microbial populations is an evolutionary conundrum for which several possible solutions have been described. In one of these, metabolic policing, quorumsensing cooperators secrete an intoxicant to punish cheaters that do not produce public goods 9 . In $P$. aeruginosa, one such intoxicant is cyanide. Because cyanide production is QS-regulated, a reasonable assumption would be that QS regulates both the production of cyanide and the necessary enzymes for detoxification. However, previously published studies of QS-regulated genes have not identified candidates for this function. In the present work, we demonstrated that QS cooperators are more resistant to cyanide intoxication than QS-null LasR mutants, which are cheaters under the conditions of our experiments (Fig. 1a). The WT is also more resistant to damage from the generation of ROS than the LasR mutant (Fig. 1b).

We demonstrated that the regulation of genes encoding the cyanide-insensitive terminal oxidase Cio is involved in protection of cooperators from cyanide intoxication, and that $\mathrm{cio} A B$ is coinduced by QS and cyanide (Fig. 2a). This feature of QS gene expression by AND gates is characteristic of $P$. aeruginosa $a^{25}$, and explains why $\operatorname{cio} A B$ were not identified as QS regulated in prior transcriptomic studies ${ }^{12,13}$.

While expression of genes in the $h c n A B C$ operon have been found to be robustly QS-controlled in multiple growth media and at multiple stages of growth ${ }^{12,13,26}$, translation and resulting 
elaboration of measurable cyanide by QS-proficient strains is delayed until cells are firmly in stationary phase ${ }^{27}$, further stalling cyanide-dependent induction of $\operatorname{cio} A B$ and preventing discovery of these genes as QS-dependent. The mechanism by which cyanide concentrations in the environment or the resulting ecological effects are sensed and integrated into the QS-dependent transcriptional program of $P$. aeruginosa remains elusive.

Other candidates for QS-regulated cyanide detoxification, including the gene products of PA4129-4134 and $r h d A$, were not involved under the conditions of our experiments and $r h d A$ was not expressed (Fig. 2b). However, our data do not exclude a role for the products of these genes in $P$. aeruginosa cyanide detoxification; they are merely not the key players in QS-mediated resistance to policing. In particular, we show that PA4133 is, like cio $A B$, conditionally regulated by QS when cyanide is present. There may be other circumstances where cyanide production is important, including in interspecific competition ${ }^{28}$, where the contribution of these other factors may be comparatively greater.

We also showed that supplementation of the growth media with copper sulfate, which alters the efficiency of non-Cio cytochrome oxidases $^{29}$, or $\mathrm{N}$-acetylcysteine, an ROS scavenger, has the potential to induce the collapse of a mixed population of $P$. aeruginosa. We suspect that they do so by reducing the difference in cyanide susceptibility between the WT and LasR mutants, pointing to a potential use for this strategy in settings where a population collapse of $P$. aeruginosa would be desirable. When cooperators are no longer able to selectively intoxicate cheaters effectively, in addition to the metabolic cost of cooperative protease secretion, they suffer the metabolic cost of policing without a commensurate benefit, and cheaters rise to an unsustainable frequency (Fig. 4d).

In a setting where policing is the major restraint on cheaters, there would be a selective pressure for cheaters to develop a nonQS-regulated mechanism to resist cyanide toxicity. This might take the form of constitutive expression of $\operatorname{cio} A B$, upregulation of one of the other genes involved in cyanide detoxification, or by some other mechanism. However, even in 30 -day passages ${ }^{3,4}$ such variants did not appear to emerge. It may be that the cost to the cheater of this kind of a regulatory rewiring would not exceed the benefit, as the selective pressure (at least in the conditions of our experiments) is not very strong: even with policing, cheaters still come to comprise $\sim 20 \%$ of the population.

Our work illustrates the intricacy of QS regulation in P. aeruginosa and the conditional nature of some elements of the regulon. This kind of regulation might allow bacterial populations to behave differently depending on environmental cues including, in this case, the presence of cheaters. For applications where a loss of quorum sensing induction in the population may be beneficial, such as in acute infections or biofouling ${ }^{30-32}$, our work points to new avenues of development for biological control of $P$. aeruginosa.

\section{Methods}

Bacterial strains and growth conditions. Bacterial strains used in this study are listed in Supplementary Table 1. Bacteria were grown in minimal medium with $1 \%$ (w/v) sodium caseinate as the sole carbon source ("casein broth") ${ }^{4}$ or in LB buffered with $20 \mathrm{mM} \mathrm{N}$-morpholinopropanesulfonic acid (LB-MOPS) ${ }^{33}$. Unless otherwise specified, cultures were grown in 16-mm test tubes containing $4 \mathrm{ml}$ media, with shaking $(225 \mathrm{RPM})$ at $37^{\circ} \mathrm{C}$. Cell densities in LB-MOPS were determined as the optical density at $600 \mathrm{~nm}$ using a spectrophotometer.

All mutants used in this study were generated using homologous recombination as previously described ${ }^{34}$. PCR-amplified DNA fragments flanking cioA, PA4133, and $r h d A$ were cloned into pEXG2 ${ }^{34,35}$. Briefly, Escherichia coli S17-1 containing pEXG2 constructs were mated with PAO1. Transconjugants were selected on Pseudomonas Isolation Agar (PIA) containing gentamicin. Deletion mutants were selected on PIA containing 5\% (w/v) sucrose. Mutants were confirmed by PCR of genomic DNA.

The arabinose-inducible $h c n A B C$ construct was generated using E. colimediated homologous recombination as previously described ${ }^{36}$. Fragments encompassing the araC gene and ParaBAD promoter, the hon $A B C$ operon, and the
pUC18T-miniTn7T-GmR backbone were PCR-amplified using primers with homologous ends from pJN105 plasmid DNA, PAO1 genomic DNA, and pUC18T-miniTn7T-GmR plasmid DNA, respectively. The complete plasmid construct was then electroporated, along with helper plasmid pTNS $2^{37}$, into PAO1 and the LasR-null mutant to facilitate insertion at the att site in each strain. Transformants were selected on LB agar containing gentamicin and confirmed by PCR.

Bacterial resistance to $\mathbf{C N}^{-}$or $\mathbf{H}_{\mathbf{2}} \mathbf{O}_{2}$. Fresh colonies of $P$. aeruginosa PAO1, LasR-null, PA4133-nul, RhdA-null, or CioA-null strains were inoculated into $4 \mathrm{ml}$ of LB-MOPS. From these initial cultures, $0.4 \mathrm{ml}$ was subcultured into $4 \mathrm{ml}$ fresh LB-MOPS, and grown to mid-log phase $\left(\mathrm{OD}_{600}\right.$ of 0.5$)$. We inoculated $40 \mu \mathrm{l}$ from these cultures into $4 \mathrm{ml}$ of fresh LB-MOPS (an initial $\mathrm{OD}_{600}$ of 0.005 ) in the presence of $0-500 \mu \mathrm{M} \mathrm{KCN}$ or $0-15 \mathrm{mM} \mathrm{H}_{2} \mathrm{O}_{2}$. We then calculated a survival ratio: the number of cells in the treated groups divided by the number in the untreated group. All experiments were performed in five replicates.

Competition experiments. We combined two strains at an initial $\mathrm{OD}_{600}$ of 0.05 in casein broth. The initial ratio of cooperators (PAO1, CioA-null or PA4133-null) to LasR-null was 99:1, as determined by $\mathrm{OD}_{600}$ from overnight growth in LB-MOPS. In some experiments, $25 \mathrm{U} / \mathrm{ml}$ catalase was added to casein broth. At $24 \mathrm{~h}$ intervals, we transferred $50 \mu \mathrm{l}$ to fresh casein broth with or without catalase. Periodically, cells were plated for isolated colonies on LB agar. Cheater abundance was determined by patching colonies on skim milk agar, as previously described ${ }^{4}$; we enumerated at least 100 colonies for each condition at each timepoint.

Measurement of ROS, $\mathbf{C N}^{-}$, and elastase. Reactive oxygen species were detected using a $2^{\prime}, 7^{\prime}$-dichlorofluorescein diacetate (DCFH-DA) probe ${ }^{24}$. Briefly, DCFHDA was added to cultures at a final concentration of $10 \mu \mathrm{M}$. After incubation in the dark at $37^{\circ} \mathrm{C}$ for $30 \mathrm{~min}$, cells were washed with PBS to thoroughly remove extracellular DCFH-DA. The reaction of DCFH-DA with ROS generates a fluorescent, oxidized derivative, DCF (F). Individual cell fluorescence was measured using a CytoFlex S (Beckman Coutler, USA) flow cytometer in the FITC channel. Events were analyzed using CytExpert 2.0 software (Beckman Coulter). As the experiments were monocultures grown in LB-MOPS, all events were counted (i.e., there was no exclusion gate). Alternatively, total DCF (F) levels were measured in a SpectraMax ${ }^{\circledast}$ i3 plate reader (Molecular Devices, USA) (488 nm excitation, $525 \mathrm{~nm}$ detection). The relative ROS level was calculated by dividing the DCF(F) level by that of PBS.

Cyanide concentrations were determined using a $\mathrm{CN}^{-}$ion selective electrode (Cole-Parmer, USA). Briefly, the overnight culture was adjusted to $\mathrm{pH} 12.0$ with $\mathrm{NaOH}$, and then $\mathrm{CN}^{-}$ion detected by conductivity. A standard curve of $\mathrm{KCN}$ was made for calculation of the $\mathrm{CN}^{-}$concentrations in samples.

Elastase production was measured using the Pierce Fluorescent Protease Assay kit (Thermo, USA). Briefly, the culture was centrifuged at $15,000 \mathrm{~g}$ for $5 \mathrm{~min}$. A total of $100 \mu \mathrm{l}$ of supernatant was mixed with $100 \mu \mathrm{l}$ of FTC-Casein working reagent, and incubated for $45-60 \mathrm{~min}$ in the dark at room temperature. The fluorescence at $450 \mathrm{~nm}$ was detected using a SpectraMax ${ }^{\circledast}$ i3 plate reader (Molecular Devices, USA).

RT-qPCR. Cells were pelleted by centrifugation and RNA isolation and cDNA synthesis were performed as described elsewhere ${ }^{38}$. One microliter of cDNA was used as the template in quantitative PCR (qPCR) amplification with designed primers (Supplementary Table 2). After 40 cycles $\left(94^{\circ} \mathrm{C}\right.$ for $10 \mathrm{~min}, 94^{\circ} \mathrm{C}$ for $5 \mathrm{~s}$, $55^{\circ} \mathrm{C}$ for $15 \mathrm{~s}, 72^{\circ} \mathrm{C}$ for $10 \mathrm{~s}$ ), a melting curve analysis was performed $\left(60-95^{\circ} \mathrm{C}\right)$ to verify the specificity of amplicons. The relative level of expression $(Q)$ for a given gene was calculated based on the formula $Q=2^{-\Delta C t}$. Each Ct value represents the average of three replicates, where $\Delta \mathrm{Ct}$ represents the target gene $\mathrm{Ct}$ minus the $\mathrm{Ct}$ of the housekeeping gene proc $^{39}$.

Reporting summary. Further information on research design is available in the Nature Research Reporting Summary linked to this article.

\section{Data availability}

The datasets generated and analysed during the current study are available in the accompanying Source Data file. The strains used in these studies are available from the corresponding authors on reasonable request.

Received: 12 December 2018; Accepted: 16 October 2019; Published online: 01 November 2019

\section{References}

1. Schuster, M. et al. Acyl-homoserine lactone quorum sensing: from evolution to application. Annu. Rev. Microbiol. 67, 43-63 (2013). 
2. Papenfort, K. et al. Quorum sensing signal-response systems in Gram-negative bacteria. Nat. Rev. Microbiol. 14, 576-588 (2016).

3. Sandoz, K. et al. Social cheating in Pseudomonas aeruginosa quorum sensing. Proc. Natl Acad. Sci. USA 104, 15876-15881 (2007).

4. Dandekar, A. A. et al. Bacterial quorum sensing and metabolic incentives to cooperate. Science 338, 264-266 (2012).

5. Diggle, S. P. et al. Cooperation and conflict in quorum-sensing bacterial populations. Nature 450, 411-414 (2007).

6. Asfahl, K. L. et al. Social interactions in bacterial cell-cell signaling. FEMS Microbiol. Rev. 41, 92-107 (2017).

7. Xavier, J. B. et al. A molecular mechanism that stabilizes cooperative secretions in Pseudomonas aeruginosa. Mol. Microbiol. 79, 166-179 (2011).

8. Özkaya, Ö. et al. Cheating on cheaters stabilizes cooperation in Pseudomonas aeruginosa. Curr. Biol. 28, 2070-2080 (2018).

9. Wang, M. et al. Quorum sensing and policing of Pseudomonas aeruginosa social cheaters. Proc. Natl Acad. Sci. USA 112, 2187-2191 (2015).

10. Pessi, G. et al. Transcriptional control of the hydrogen cyanide biosynthetic genes $h c n A B C$ by the anaerobic regulator ANR and the quorum-sensing regulators LasR and RhlR in Pseudomonas aeruginosa. J. Bacteriol. 182, 6940-6949 (2000).

11. Anderson, M. S. et al. Kind discrimination and competitive exclusion mediated by contact-dependent growth inhibition systems shape biofilm community structure. PLoS Pathog. 10, e1004076 (2014).

12. Whiteley, M. et al. Identification of genes controlled by quorum sensing in Pseudomonas aeruginosa. Proc. Natl Acad. Sci. USA 96, 13904-13909 (1999).

13. Schuster, M. et al. Identification, timing, and signal specificity of Pseudomonas aeruginosa quorum-controlled genes: a transcriptome analysis. J. Bacteriol. 185, 2066-2079 (2003).

14. Gilchrist, F. J. et al. Variation in hydrogen cyanide production between different strains of Pseudomonas aeruginosa. Eur. Respir. J. 38, 409-414 (2011).

15. Bernier, S. P. et al. Cyanide toxicity to Burkholderia cenocepacia is modulated by polymicrobial communities and environmental factors. Front. Microbiol. 7, 725 (2016).

16. Hazan, R. et al. Auto poisoning of the respiratory chain by a quorum-sensingregulated molecule favors biofilm formation and antibiotic tolerance. Curr. Biol. 26, 195-206 (2016).

17. Cunningham, L. et al. The cioAB genes from Pseudomonas aeruginosa code for a novel cyanide-insensitive terminal oxidase related to the cytochrome bd quinol oxidases. Mol. Microbiol. 24, 579-591 (1997).

18. Cipollone, R. et al. Involvement of Pseudomonas aeruginosa rhodanese in protection from cyanide toxicity. Appl. Environ. Microbiol. 73, 390-398 (2007).

19. Frangipani, E. et al. A novel cyanide-inducible gene cluster helps protect Pseudomonas aeruginosa from cyanide. Environ. Microbiol. Rep. 6, 28-34 (2014).

20. Pessi, G. et al. Dual control of hydrogen cyanide biosynthesis by the global activator GacA in Pseudomonas aeruginosa PAO1. FEMS Microbiol. Lett. 200, 73-78 (2001)

21. Heurlier, K. et al. Negative control of quorum sensing by RpoN (sigma54) in Pseudomonas aeruginosa PAO1. J. Bacteriol. 185, 2227-2235 (2003).

22. He, X. et al. Autoinduced AND gate controls metabolic pathway dynamically in response to microbial communities and cell physiological state. ACS Synth. Biol. 6, 463-470 (2017).

23. Kawakami, T. et al. Differential expression of multiple terminal oxidases for aerobic respiration in Pseudomonas aeruginosa. Environ. Microbiol. 12, 1399-1412 (2010).

24. Yan, H. et al. A metabolic trade-off modulates policing of social cheaters in populations of Pseudomonas aeruginosa. Front. Microbiol. 9, 337 (2018).

25. Schuster, M. et al. The Pseudomonas aeruginosa RpoS regulon and its relationship to quorum sensing. Mol. Microbiol. 51, 973-985 (2004).

26. Asfahl, K. L. et al. Additive effects of quorum sensing anti-activators on virulence traits and transcriptome. Front. Microbiol. 8, 2654 (2017).

27. Neerincx, A. H. et al. Real-time monitoring of hydrogen cyanide (HCN) and ammonia $\left(\mathrm{NH}_{3}\right)$ emitted by Pseudomonas aeruginosa. J. Breath. Res. 9, 027102 (2015).

28. Abisado, R. et al. Bacterial quorum sensing and microbial community interactions. mBio 9, e02331-17 (2017).

29. Hirai, T. et al. Expression of multiple cytochrome oxidase isoforms by combinations of multiple isosubunits in Pseudomonas aeruginosa. Proc. Natl Acad. Sci. USA 113, 12815-12819 (2016).
30. Shrout, J. et al. Monitoring bacterial twitter: does quorum sensing determine the behavior of water and wastewater treatment biofilms? Environ. Sci. Technol. 46, 1995-2005 (2012).

31. Curran, C. S. et al. Mechanisms and targeted therapies for Pseudomonas aeruginosa lung infection. Am. J. Respir. Crit. Care. Med. 197, 708-727 (2018).

32. Kampouris, I. D. et al. Evaluation of a novel quorum quenching strain for MBR biofouling mitigation. Water Res. 143, 56-65 (2018).

33. Sambrook, J. et al. Molecular Cloning: A Laboratory Manual (Cold Spring Harbor Laboratory Press, Plainview, NY, 1989).

34. Rietsch, A. et al. ExsE, a secreted regulator of type III secretion genes in Pseudomonas aeruginosa. Proc. Natl Acad. Sci. USA 102, 8006-8011 (2005).

35. Stover, C. K. et al. Complete genome sequence of Pseudomonas aeruginosa PAO1, an opportunistic pathogen. Nature 406, 959-964 (2000).

36. Kostylev, M. et al. Cloning should be simple: Escherichia coli DH5a-mediated assembly of multiple DNA fragments with short end homologies. PLoS. One. 10, e0137466 (2015)

37. Choi, K. H. et al. mini-Tn7 insertion in bacteria with secondary, non-glmSlinked attTn7 sites: example Proteus mirabilis HI4320. Nat. Protoc. 1, 170-178 (2006).

38. Chomczynski, P. et al. The single-step method of RNA isolation by acid guanidinium thiocyanate-phenol-chloroform extraction: twenty-something years on. Nat. Protoc. 1, 581 (2006).

39. Zhang, Y. Y. et al. Interference of non-lethal levels of graphene oxide in biofilm formation and adaptive response of quorum sensing in bacteria. Environ. Sci.: Nano. 5, 2809-2818 (2018).

\section{Acknowledgements}

We thank E.P. Greenberg for reviewing the manuscript. This work was funded by National Key R\&D Program of China (Grant no. 2018YFE0110500), and the National Science Foundation of China (Grant nos. 31570490 and 21876155). AAD received support from the Burroughs-Wellcome Fund (1012253), and KLA from the US National Institutes of Health (T32 HL007287).

\section{Author contributions}

H.Y., D.S., K.L.A, A.A.D. and M.W. designed research; H.Y., N.L., F.S., J.X. and K.L.A. performed research; H.Y., K.L.A., A.A.D. and M.W. analyzed data; and H.Y., K.L.A., A.A.D. and M.W. wrote the paper.

\section{Competing interests}

The authors declare no competing interests.

\section{Additional information}

Supplementary information is available for this paper at https://doi.org/10.1038/s41467 019-13013-8.

Correspondence and requests for materials should be addressed to A.A.D. or M.W.

Peer review information Nature Communications thanks the anonymous reviewers for their contribution to the peer review of this work.

Reprints and permission information is available at http://www.nature.com/reprints

Publisher's note Springer Nature remains neutral with regard to jurisdictional claims in published maps and institutional affiliations.

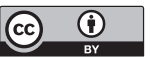

Open Access This article is licensed under a Creative Commons Attribution 4.0 International License, which permits use, sharing, adaptation, distribution and reproduction in any medium or format, as long as you give appropriate credit to the original author(s) and the source, provide a link to the Creative Commons license, and indicate if changes were made. The images or other third party material in this article are included in the article's Creative Commons license, unless indicated otherwise in a credit line to the material. If material is not included in the article's Creative Commons license and your intended use is not permitted by statutory regulation or exceeds the permitted use, you will need to obtain permission directly from the copyright holder. To view a copy of this license, visit http://creativecommons.org/ licenses/by/4.0/.

(C) The Author(s) 2019 